\title{
ON THE EDGE COLORING OF GRAPH PRODUCTS
}

\author{
M. M. M. JARADAT
}

Received 11 October 2004

The edge chromatic number of $G$ is the minimum number of colors required to color the edges of $G$ in such a way that no two adjacent edges have the same color. We will determine a sufficient condition for a various graph products to be of class 1, namely, strong product, semistrong product, and special product.

\section{Introduction}

All graphs under consideration are nonnull, finite, undirected, and simple graphs. We adopt the standard notations $d_{G}(v)$ for the degree of the vertex $v$ in the graph $G$, and $\Delta(G)$ for the maximum degree of the vertices of $G$.

The edge chromatic number, $\chi^{\prime}(G)$, of $G$ is the minimum number of colors required to color the edges of $G$ in such a way that no two adjacent edges have the same color. A graph is called a $k$-regular graph if the degree of each vertex is $k$. A cycle of a graph $G$ is said to be Hamiltonian if it passes by all the vertices of $G$. A sequence $F_{1}, F_{2}, \ldots, F_{n}$ of pairwise edge disjoint graphs with union $G$ is called a decomposition of $G$ and we write $G=\bigcup_{i=1}^{n} F_{i}$. In addition, if the subgraphs $F_{i}$ are $k$-regular spanning of $G$, then $G$ is called a $k$-factorable graph and each $F_{i}$ is called a $k$-factor. Moreover, if $F_{i}$ is Hamiltonian cycle for each $i=1,2, \ldots, n$, then $G$ is called a Hamiltonian decomposable graph. A graph $M$ is a matching if $\Delta(M)=1$, and a perfect matching if the degree of each vertex is 1 . An independent set of edges is a subset of $E(G)$ in which no two edges are adjacent. Vizing [8] classified graphs into two classes, 1 and 2; a graph $G$ is of class 1 if $\chi^{\prime}(G)=\Delta(G)$, and of class 2 if $\chi^{\prime}(G)=\Delta(G)+1$. It is known that a bipartite graph is of class 1 . Also, $a 2 r$ regular graph is 2 -factorable. It is elementary from the definitions that a graph is regular and of class 1 if and only if it is 1 -factorable.

Let $G=(V(G), E(G))$ and $H=(V(H), E(H))$ be two graphs.

(1) The direct product $G \wedge H$ has vertex set $V(G \wedge H)=V(G) \times V(H)$ and edge set $E(G \wedge H)=\left\{\left(u_{1}, v_{1}\right)\left(u_{2}, v_{2}\right) \mid u_{1} u_{2} \in E(G)\right.$ and $\left.v_{1} v_{2} \in E(H)\right\}$.

(2) The Cartesian product $G \times H$ has vertex set $V(G \times H)=V(G) \times V(H)$ and edge set $E(G \times H)=\left\{\left(u_{1}, v_{1}\right)\left(u_{2}, v_{2}\right) \mid u_{1} u_{2} \in E(G)\right.$ and $v_{1}=v_{2}$, or $u_{1}=u_{2}$ and $v_{1} v_{2} \in$ $E(H)\}$. 
(3) The strong product $G \otimes H$ has vertex set $V(G \otimes H)=V(G) \times V(H)$ and edge set $E(G \otimes H)=\left\{\left(u_{1}, v_{1}\right)\left(u_{2}, v_{2}\right) \mid u_{1} u_{2} \in E(G)\right.$ and $v_{1} v_{2} \in E(H)$ or $u_{1} u_{2} \in E(G)$ and $v_{1}=v_{2}$, or $u_{1}=u_{2}$ and $\left.v_{1} v_{2} \in E(H)\right\}$.

(4) The semistrong product $G \bullet H$ has vertex set $V(G \bullet H)=V(G) \times V(H)$ and edge set $E(G \bullet H)=\left\{\left(u_{1}, v_{1}\right)\left(u_{2}, v_{2}\right) \mid u_{1} u_{2} \in E(G)\right.$ and $v_{1} v_{2} \in E(H)$, or $u_{1}=u_{2}$ and $\left.v_{1} v_{2} \in E(H)\right\}$.

(5) The lexicographic product $G[H]$ has vertex set $V(G[H])=V(G) \times V(H)$ and edge set $E(G[H])=\left\{\left(u_{1}, v_{1}\right)\left(u_{2}, v_{2}\right) \mid u_{1} u_{2} \in E(G)\right.$, or $u_{1}=u_{2}$ and $\left.v_{1} v_{2} \in E(H)\right\}$.

(6) The special product $G \oplus H$ has vertex set $V(G \oplus H)=V(G) \times V(H)$ and edge set $E(G \oplus H)=\left\{\left(u_{1}, v_{1}\right)\left(u_{2}, v_{2}\right) \mid u_{1} u_{2} \in E(G)\right.$ or $\left.v_{1} v_{2} \in E(H)\right\}$.

(7) The wreath product $G \rho H$ has vertex set $V(G \rho H)=V(G) \times V(H)$ and edge set $E(G \rho H)=\left\{\left(u_{1}, v_{1}\right)\left(u_{2}, v_{2}\right) \mid u_{1}=u_{2}\right.$ and $v_{1} v_{2} \in E(H)$, or $u_{1} u_{2} \in E(G)$ and there is $\alpha \in \operatorname{Aut}(H)$ such that $\left.\alpha\left(v_{1}\right)=v_{2}\right\}$, where $\operatorname{Aut}(H)$ is the automorphism group of $H$. Note that

$$
d_{G \oplus H}(u, v)=d_{G}(u)|V(H)|+d_{H}(v)|V(G)|-d_{H}(v) d_{G}(u) .
$$

For a long time, the question of whether the product of two graphs is of class 1 , if one of the graphs is of class 1 , has been studied by a number of authors. The following theorem, due to Mahmoodian [6], answers the question for the Cartesian product.

Theorem 1.1 (E. S. Mahmoodian). Let $G^{*}=G \times H$ be the Cartesian product of $G$ and $H$. If one of $G$ and $H$ is of class 1 , then $G^{*}$ is of class 1 .

The (noncommutative) lexicographic product has been studied by Anderson and Lipman [1], Pisanski et al. [7], and Jaradat [4].

Theorem 1.2 (Anderson and Lipman). Let $G$ and $H$ be two graphs. If $G$ is of class 1 , then $G[H]$ is of class 1 .

Theorem 1.3 (Jaradat). Let $G$ and $H$ be two graphs. If $\chi^{\prime}(H)=\Delta(H)$ and $H$ is of even order, then $\chi^{\prime}(G[H])=\Delta(G[H])$. Moreover, the corresponding statement needs not hold when $H$ has odd order.

The (noncommutative) wreath product has been studied by Anderson and Lipman [1] and Jaradat [4] who proved the following.

Theorem 1.4 (Anderson and Lipman). Let $G$ be of class 1. If $H$ has the property that a vertex in the largest isomorphism class of vertices in $H$ has the maximum degree in $H$, then $\mathrm{G} \rho \mathrm{H}$ is of class 1 .

Anderson and Lipman conjectured that if $G$ is of class 1, then $G \rho H$ is of class 1 . The same conjecture appeared in Jensen and Toft's book [5] as a question. The next result due to Jaradat [4] is a major progress to the conjecture, there are still some cases unsettled.

Theorem 1.5 (Jaradat). Let $G$ and $H$ be two graphs such that $G$ is of class 1. Then, $G \rho H$ is of class 1 if one of the following holds: (i) $\chi^{\prime}(H)-\delta(H) \leq \Delta(G)$, (ii) $\Delta(H)=\Delta(G)$, (iii) $\Delta(H)<2 \Delta(G)$, and $\left|\left\{v \in V(H): d_{H}(v)=0\right\}\right|>|V(H)| / 2$. 
Also, Anderson and Lipman posed the question about the edge chromatic number of $G \rho P_{2}$ when $G$ is of class 2 and hinted that this would be a difficult problem. Jaradat gave a complete answer for this question when he proved a more general case as in the following result.

Theorem 1.6 (Jaradat). Let $G$ and $H$ be two graphs. If $H$ is vertex-transitive of even order, and if $\chi^{\prime}(H)=\Delta(H)$, then $\chi^{\prime}(G \rho H)=\Delta(G \rho H)$.

The direct product has been studied by Jaradat who proved the following result.

Theorem 1.7 (Jaradat). Let $G$ and $H$ be two graphs such that at least one of them is of class 1 , then $G \wedge H$ is of class 1 .

In this paper, we determine sufficient condition for various graph products to be of class 1, namely, strong product, semistrong product, and special product of two graphs.

\section{Main results}

We start this section by focusing on the chromatic number of the strong product of two graphs. Note that $\Delta(G \otimes H)=\Delta(G)+\Delta(H)+\Delta(G) \Delta(H)$.

Theorem 2.1. Let $G$ and $H$ be two graphs such that at least one of them is of class 1 , then $G \otimes H$ is of class 1 .

Proof. It is an easy matter to see that $G \otimes H=(G \times H) \cup(G \wedge H)$. And so, $\chi^{\prime}(G \otimes H) \leq$ $\chi^{\prime}(G \times H)+\chi^{\prime}(G \wedge H)$. Since at least one of $G$ and $H$ is of class 1, by Theorems 1.1 and 1.7, $\chi^{\prime}(G \times H) \leq \Delta(G)+\Delta(H)$ and $\chi^{\prime}(G \wedge H) \leq \Delta(G) \Delta(H)$. Therefore, $\chi^{\prime}(G \otimes H) \leq \Delta(G)+$ $\Delta(H)+\Delta(G) \Delta(H)=\Delta(G \otimes H)$. The proof is complete.

The following result is a straightforward consequence of Theorem 2.1 and the fact that a regular graph is of class 1 if and only if it is 1 -factorable.

Corollary 2.2 (Zhou). Let $G$ and $H$ be two graphs such that at least one of them is 1factorable and the other is regular, then $G \otimes H$ is 1-factorable.

Now, we turn our attention to deal with the chromatic number of the semistrong product of graphs. Note that $\Delta(G \bullet H)=\Delta(G) \Delta(H)+\Delta(H)$.

Lemma 2.3. Let $H$ be a $2 r$-regular graph and let $M$ be a matching, then $\chi^{\prime}(M \bullet H)=4 r$.

Proof. Since $H$ is a $2 r$-regular graph, $H$ is a 2-factorable graph, say, $H=\cup_{i=1}^{r} C_{i}^{*}$. And so, $C_{i}^{*}$ is decomposable into vertex disjoint union of cycles, say, $C_{i}^{*}=\cup_{j=1}^{j_{i}} C_{i}^{(j)}$. Since $M$ is a matching, $M$ is decomposable into a vertex disjoint union of $\left\{K_{2}^{(f)}\right\}_{f=1}^{l} \cup\left\{u_{t}\right\}_{t=1}^{s}$, where $K_{2}^{(f)}$ is a complete graph of order 2 and $u_{t}$ is an isolated vertex. Therefore,

$$
\begin{aligned}
M \bullet H & =\left(\left(\bigcup_{f=1}^{l} K_{2}^{(f)}\right) \bigcup\left(\bigcup_{t=1}^{s} u_{t}\right)\right) \bullet H \\
& =\left(\bigcup_{f=1}^{l}\left(K_{2}^{(f)} \bullet H\right)\right) \bigcup\left(\bigcup_{t=1}^{s}\left(u_{t} \times H\right)\right)
\end{aligned}
$$




$$
\begin{aligned}
& =\bigcup_{i=1}^{r}\left(\bigcup_{f=1}^{l}\left(K_{2}^{(f)} \bullet C_{i}^{*}\right)\right) \bigcup\left(\bigcup_{t=1}^{s}\left(u_{t} \times H\right)\right) \\
& =\bigcup_{i=1}^{r}\left(\bigcup_{f=1}^{l}\left(\bigcup_{j=1}^{j_{i}}\left(K_{2}^{(f)} \bullet C_{i}^{(j)}\right)\right)\right) \bigcup\left(\bigcup_{t=1}^{s}\left(u_{t} \times H\right)\right) .
\end{aligned}
$$

Since $K_{2}^{(f)} \bullet C_{i}^{(j)}$ is Hamiltonian decomposable into two even cycles, as a result $\chi^{\prime}\left(K_{2}^{(f)} \bullet\right.$ $\left.C_{i}^{(j)}\right)=4$. Since no vertex of $C_{i}^{(j)}$ is adjacent to a vertex of $C_{i}^{(k)}$, we have that no vertex of $K_{2}^{(f)} \bullet C_{i}^{(j)}$ is adjacent to a vertex of $K_{2}^{(f)} \bullet C_{i}^{(k)}$, whenever $j \neq k$. Thus, $\chi^{\prime}\left(K_{2}^{(f)} \bullet C_{i}^{*}\right)=$ $\chi^{\prime}\left(\bigcup_{j=1}^{j_{i}}\left(K_{2}^{(f)} \bullet C_{i}^{(j)}\right)\right)=4$. Also, since no vertex of $K_{2}^{(f)}$ is adjacent to a vertex of $K_{2}^{(h)}$, it implies that no vertex of $\bigcup_{j=1}^{j_{i}}\left(K_{2}^{(f)} \bullet C_{i}^{(j)}\right)$ is adjacent to a vertex of $\bigcup_{j=1}^{j_{i}}\left(K_{2}^{(h)} \bullet C_{i}^{(j)}\right)$ for any $f \neq h$. Thus, $\chi^{\prime}\left(\bigcup_{f=1}^{l} \bigcup_{j=1}^{j_{i}}\left(K_{2}^{(f)} \bullet C_{i}^{(j)}\right)\right)=\chi^{\prime}\left(\bigcup_{j=1}^{j_{i}}\left(K_{2}^{(f)} \bullet C_{i}^{(j)}\right)\right)=4$. Since $\left\{u_{t} \times\right.$ $H\}_{t=1}^{s}$ is a set of disjoint copies of $H$, and since $\chi^{\prime}(H) \leq 2 r+1$, we have that $\chi^{\prime}\left(\bigcup_{t=1}^{s}\left(u_{t} \times\right.\right.$ $H))=\chi^{\prime}\left(u_{t} \times H\right)=\chi^{\prime}(H) \leq 2 r+1$. Finally, no vertex of $\bigcup_{i=1}^{r}\left(\bigcup_{f=1}^{l}\left(\bigcup_{j=1}^{j_{i}}\left(K_{2}^{(f)} \bullet C_{i}^{(j)}\right)\right)\right)$ is adjacent to a vertex of $\bigcup_{t=1}^{s}\left(u_{t} \times H\right)$. Therefore,

$$
\begin{aligned}
\chi^{\prime}\left(M_{1} \bullet H\right) & \leq \max \left\{\sum_{i=1}^{r} \chi^{\prime}\left(\bigcup_{f=1}^{l} \bigcup_{j=1}^{j_{i}}\left(K_{2}^{(f)} \bullet C_{i}^{(j)}\right)\right), \chi^{\prime}(H)\right\} \\
& \leq \max \{4 r, 2 r+1\} \\
& =4 r .
\end{aligned}
$$

The proof is complete.

Lemma 2.4. Let $K_{2}$ be a path of order 2 and $M$ be a perfect matching, then $K_{2} \bullet M$ is a bipartite graph and so $\chi^{\prime}\left(K_{2} \bullet M\right)=2$.

Proof. The proof follows by noting that $K_{2} \bullet M$ is decomposable into vertex disjoint cycles of order 4 . The proof is complete.

Lemma 2.5. Let $H$ be a $(2 r+1)$-regular graph having 1 -factor and let $M$ be a matching, then $\chi^{\prime}(M \bullet H)=4 r+2$.

Proof. Let $M_{H}$ be a 1 -factor of $H$, then $H-M_{H}$ is a $2 r$-regular graph. Thus, $H=(H-$ $\left.M_{H}\right) \cup M_{H}$. Therefore, $M \bullet H=\left(M \bullet\left(H-M_{H}\right)\right) \cup\left(M \bullet M_{H}\right)$. By Lemma 2.3, $\chi^{\prime}(M \bullet(H-$ $\left.\left.M_{H}\right)\right)=4 r$. We now show that $\chi^{\prime}\left(M \bullet M_{H}\right)=2$. As in Lemma 2.3, $M$ is decomposable into a vertex disjoint union of $\left\{K_{2}^{(f)}\right\}_{k=1}^{l} \cup\left\{u_{t}\right\}_{t=1}^{s}$, where $K_{2}^{(f)}$ is a complete graph of order 2 and $u_{t}$ is an isolated vertex. Therefore,

$$
\begin{aligned}
M \bullet M_{H} & =\left(\left(\bigcup_{f=1}^{l} K_{2}^{(f)}\right) \bigcup\left(\bigcup_{t=1}^{s} u_{t}\right)\right) \cdot M_{H} \\
& =\left(\bigcup_{f=1}^{l}\left(K_{2}^{(f)} \bullet M_{H}\right)\right) \bigcup\left(\bigcup_{t=1}^{s}\left(u_{t} \times M_{H}\right)\right) .
\end{aligned}
$$


Since $\chi^{\prime}\left(M_{H}\right)=1$, as in Lemma 2.3, $\chi^{\prime}\left(\bigcup_{t=1}^{s}\left(u_{t} \times M_{H}\right)\right)=\chi^{\prime}\left(u_{t} \times M_{H}\right)=\chi^{\prime}\left(M_{H}\right)=1$. Clearly that, no vertex of $K_{2}^{(f)} \bullet M_{H}$ is adjacent to a vertex of $K_{2}^{(h)} \bullet M_{H}$ for any $f \neq h$. Therefore, by Lemma 2.4, $\chi^{\prime}\left(\bigcup_{f=1}^{l}\left(K_{2}^{(f)} \bullet M_{H}\right)\right)=\chi^{\prime}\left(K_{2}^{(f)} \bullet M_{H}\right)=2$. Finally, no vertex of $\bigcup_{f=1}^{l}\left(K_{2}^{(f)} \bullet M_{H}\right)$ is adjacent to any vertex of $\bigcup_{t=1}^{r}\left(u_{t} \times M_{H}\right)$. Hence, $\chi^{\prime}\left(M \bullet M_{H}\right) \leq 2$. Therefore, $\chi^{\prime}(M \bullet H)=4 r+2$. The proof is complete.

Theorem 2.6. Let $G$ and $H$ be two graphs, then $G \bullet H$ is of class 1 if one of the following holds: (i) $H$ is of class 1, (ii) $G$ is of class 1 and $H$ is an $r$-regular graph such that if $r$ is odd, then $H$ has 1-factor.

Proof. First, we consider (i). Note that $G \bullet H=(G \wedge H) \cup(N \times H)$, where $N$ is the null graph with vertex set $V(G)$. And so, $\chi^{\prime}(G \bullet H) \leq \chi^{\prime}(N \times H)+\chi^{\prime}(G \wedge H)$. By Theorem 1.7 and being that $N \times H$ is a vertex disjoint union copies of $H$ and $H$ is of class 1 , we have that $\chi^{\prime}(G \bullet H) \leq \Delta(H)+\Delta(H) \Delta(G)=\Delta(G \bullet H)$. Now, we consider (ii). Since $G$ is of class $1, G=\bigcup_{i=1}^{\Delta(G)} M_{i}$, where $M_{i}$ is a matching spanning subgraph of $G$. Hence,

$$
\begin{aligned}
G \bullet H & =\left(\bigcup_{i=1}^{\Delta(G)} M_{i}\right) \bullet H \\
& =\left(M_{1} \bullet H\right) \bigcup\left(\bigcup_{i=2}^{\Delta(G)}\left(M_{i} \wedge H\right)\right) .
\end{aligned}
$$

Thus,

$$
\chi^{\prime}(G \bullet H) \leq \chi^{\prime}\left(M_{1} \bullet H\right)+\sum_{i=2}^{\Delta(G)} \chi^{\prime}\left(M_{i} \wedge H\right)
$$

By Theorem 1.7,

$$
\chi^{\prime}(G \bullet H) \leq \chi^{\prime}\left(M_{1} \bullet H\right)+(\Delta(G)-1) \Delta(H)
$$

By Lemmas 2.3 and 2.5, we have that

$$
\chi^{\prime}(G \bullet H) \leq(\Delta(G)+1) \Delta(H)=\Delta(G \bullet H) .
$$

The proof is complete.

Corollary 2.7 (Zhou). Let $G$ be 1-factorable and let $H$ be r-regular such that if $r$ is odd, then $H$ has 1 -factor. Then $G \bullet H$ is 1 -factorable.

The following result is a straightforward from Theorem 2.6 and the fact that $K_{m(n)}=$ $K_{n} \bullet K_{m}$.

Corollary 2.8. The complete multipartite graph $K_{m(n)}$ is of class 1 if and only if $m n$ is even.

We now turn our attention to deal with the chromatic number of the special product of graphs. The proof of the following lemma is a straightforward exercise. 
2674 On the edge coloring of graph products

Lemma 2.9. For each $G$ and $H$, we have

$$
\Delta(G \oplus H)=\Delta(G)|V(H)|+\Delta(H)|V(G)|-\Delta(G) \Delta(H)
$$

Theorem 2.10. Let $G$ and $H$ be graphs, then $G \oplus H$ is of class 1 if at least one of the factors is of class 1 and of even order and the other is regular. Moreover, the corresponding statement needs not hold if we replace even by odd.

Proof. To prove the first part of the theorem, we may assume that $G$ is of class $1,|V(G)|=$ $2 n$, and $H$ is regular because $G \oplus H$ is isomorphic to $H \oplus G$. Note that

$$
G \oplus H=(G \times H) \cup\left(K_{2 n} \wedge H\right) \cup(G \wedge \bar{H})
$$

Thus,

$$
\chi^{\prime}(G \oplus H) \leq \chi^{\prime}(G \times H)+\chi^{\prime}\left(K_{2 n} \wedge H\right)+\chi^{\prime}(G \wedge \bar{H})
$$

By Theorems 1.1 and 1.7 and being that $G$ and $K_{2 n}$ are of class 1 , we have

$$
\begin{aligned}
\chi^{\prime}(G \oplus H) & \leq \Delta(G)+\Delta(H)+\Delta(H)(|V(G)|-1)+\Delta(\bar{H}) \Delta(G) \\
& =\Delta(G)+\Delta(H)|V(G)|+\Delta(G)(|V(H)|-\Delta(H)-1) \\
& =\Delta(H)|V(G)|+\Delta(G)|V(H)|-\Delta(G) \Delta(H) \\
& =\Delta(G \oplus H) .
\end{aligned}
$$

The second part of the theorem comes by taking $G=P_{2 n+1}$ and $H=K_{2 m+1}$, where $m, n \geq 1$ and note that

$$
|E(G \oplus H)|=(2 n m+m+n)(4 n m+2 m+2)+m(2 n-1)
$$

and the size of the largest independent edge set is less than or equal to $2 n m+n+m$. Hence,

$$
\chi^{\prime}(G \oplus H) \geq(4 n m+2 m+2)+\frac{m(2 n-1)}{2 n m+n+m} .
$$

Therefore, $\chi^{\prime}(G \oplus H)>(4 n m+2 m+2)=\Delta(G \oplus H)$. The proof is complete.

Corollary 2.11. Let $H$ and $G$ be two graphs, then $G \oplus H$ is 1-factorable if one of them is 1 -factorable and the other is regular.

We say that $\mathcal{W}=\left\{W_{1}, W_{2}, \ldots, W_{n}\right\}$ is a proper partition of $E(G)$ if $\mathcal{W}$ is a partition of $E(G)$ and $W_{i}$ is an independent set of edges for each $i=1,2, \ldots, n$. We give another sufficient condition for the special product to be of class 1 .

Theorem 2.12. Let $G$ and $H$ be two graphs such that $G$ is of class 1 and of even order. Let $\left\{V_{1}, V_{2}, \ldots, V_{\Delta(G)}\right\}$ and $\left\{U_{1}, U_{2}, \ldots, U_{|V(G)|-1}\right\}$ be proper partitions of $E(G)$ and $E\left(K_{|V(G)|}\right)$, respectively. If $V_{i} \subseteq U_{i}$ for each $i=1,2, \ldots, \Delta(G)$, then $G \oplus H$ is of class 1 . 
Proof. Assume that $V_{i}=\phi$ for each $i=\Delta(G)+1, \Delta(G)+2, \ldots,|V(G)|-1$. Then,

$$
\begin{aligned}
(G \wedge \bar{H}) \bigcup\left(K_{|V(G)|} \wedge H\right)= & \left(\left(\bigcup_{i=1}^{\Delta(G)} V_{i}\right) \wedge \bar{H}\right) \bigcup\left(\bigcup_{i=1}^{|V(G)|-1}\left(\left(\left(U_{i}-V_{i}\right) \cup V_{i}\right) \wedge H\right)\right) \\
= & \left(\left(\bigcup_{i=1}^{\Delta(G)} V_{i}\right) \wedge \bar{H}\right) \bigcup\left(\left(\bigcup_{i=1}^{\Delta(G)} V_{i}\right) \wedge H\right) \\
& \bigcup\left(\bigcup_{i=1}^{\Delta(G)}\left(\left(U_{i}-V_{i}\right) \wedge H\right)\right) \bigcup\left(\bigcup_{\Delta(G)+1}^{|V(G)|-1}\left(U_{i} \wedge H\right)\right) \\
= & \left(\left(\bigcup_{i=1}^{\Delta(G)} V_{i}\right) \wedge K_{|V(H)|}\right) \bigcup\left(\bigcup_{i=1}^{\Delta(G)}\left(\left(U_{i}-V_{i}\right) \wedge H\right)\right) \\
& \bigcup\left(\bigcup_{\Delta(G)+1}^{|V(G)|-1}\left(U_{i} \wedge H\right)\right) \\
= & \left(\bigcup_{i=1}^{\Delta(G)}\left(\left(V_{i} \wedge K_{|V(H)|}\right) \cup\left(\left(U_{i}-V_{i}\right) \wedge H\right)\right)\right) \\
& \bigcup\left(\bigcup_{\Delta(G)+1}^{|V(G)|-1}\left(U_{i} \wedge H\right)\right) .
\end{aligned}
$$

Thus, by Theorem 1.7, we have

$$
\begin{aligned}
\chi^{\prime}\left(( G \wedge \overline { H } ) \bigcup \left(K_{|V(G)| \wedge H)) \leq}\right.\right. & \chi^{\prime}\left(\bigcup_{i=1}^{\Delta(G)}\left(\left(V_{i} \wedge K_{|V(H)|}\right) \cup\left(\left(U_{i}-V_{i}\right) \wedge H\right)\right)\right) \\
& +\chi^{\prime}\left(\bigcup_{i=\Delta(G)+1}^{|V(G)|-1}\left(U_{i} \wedge H\right)\right) \\
\leq & \sum_{i=1}^{\Delta(G)} \chi^{\prime}\left(\left(V_{i} \wedge K_{|V(H)|}\right) \cup\left(\left(U_{i}-V_{i}\right) \wedge K_{|V(H)|}\right)\right) \\
& +\sum_{i=\Delta(G)+1}^{|V(G)|-1} \chi^{\prime}\left(U_{i} \wedge H\right) \\
= & \sum_{i=1}^{\Delta(G)} \chi^{\prime}\left(U_{i} \wedge K_{|V(H)|}\right)+\sum_{i=\Delta(G)+1}^{|V(G)|-1} \chi^{\prime}\left(U_{i} \wedge H\right) \\
\leq & \sum_{i=1}^{\Delta(G)}(|V(H)|-1)+\sum_{i=\Delta(G)+1} \Delta(H) \\
= & \Delta(G)(|V(H)|-1)+\Delta(H)(|V(G)|-1-\Delta(G)) \\
= & \Delta(G)|V(H)|+\Delta(H)|V(G)|-\Delta(G) \Delta(H)-\Delta(G) \\
& -\Delta(H) . \quad
\end{aligned}
$$


But as in Theorem 2.10,

$$
G \oplus H=(G \wedge \bar{H}) \bigcup\left(K_{|V(G)|} \wedge H\right) \bigcup(G \times H) .
$$

Therefore, by Theorem 1.1,

$$
\begin{aligned}
\chi^{\prime}(G \oplus H) & \leq \Delta(G)|V(H)|+\Delta(H)|V(G)|-\Delta(G) \Delta(H)-\Delta(G)-\Delta(H)+\chi^{\prime}(G \times H) \\
& =\Delta(G)|V(H)|+\Delta(H)|V(G)|-\Delta(G) \Delta(H)-\Delta(G)-\Delta(H)+\Delta(G)+\Delta(H) \\
& =\Delta(G)|V(H)|+\Delta(H)|V(G)|-\Delta(G) \Delta(H)=\Delta(G \oplus H) .
\end{aligned}
$$

The proof is complete.

\section{Acknowledgments}

I would like to thank Professor C. Y. Chao and Professor Peter Johnson for their valuable comments.

\section{References}

[1] M. Anderson and M. Lipman, The wreath product of graphs, Graphs and Applications (Boulder, Colo, 1982), John Wiley \& Sons, New York, 1985, pp. 23-39.

[2] R. Diestel, Graph Theory, Graduate Texts in Mathematics, vol. 173, Springer, New York, 1997.

[3] P. Himelwright, W. D. Wallis, and J. E. Williamson, On one-factorizations of compositions of graphs, J. Graph Theory 6 (1982), no. 1, 75-80.

[4] M. M. Jaradat, On the Anderson-Lipman conjecture and some related problems, Discrete Mathematics 297 (2005), 167-173.

[5] T. R. Jensen and B. Toft, Graph Coloring Problems, Wiley-Interscience Series in Discrete Mathematics and Optimization, John Wiley \& Sons, New York, 1995.

[6] E. S. Mahmoodian, On edge-colorability of Cartesian products of graphs, Canad. Math. Bull. 24 (1981), no. 1, 107-108.

[7] T. Pisanski, J. Shawe-Taylor, and B. Mohar, 1-factorization of the composition of regular graphs, Publ. Inst. Math. (Beograd) (N.S.) 33(47) (1983), 193-196.

[8] V. G. Vizing, On an estimate of the chromatic class of a p-graph, Diskret. Analiz. 3 (1964), 25-30 (Russian).

[9] M. K. Zhou, Decomposition of some product graphs into 1-factors and Hamiltonian cycles, Ars Combin. 28 (1989), 258-268.

M. M. M. Jaradat: Department of Mathematics, Yarmouk University, Irbid 211-63, Jordan

E-mail address: mmjst4@yu.edu.jo 


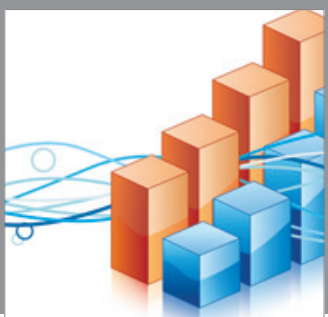

Advances in

Operations Research

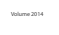

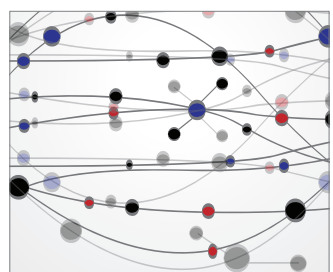

\section{The Scientific} World Journal
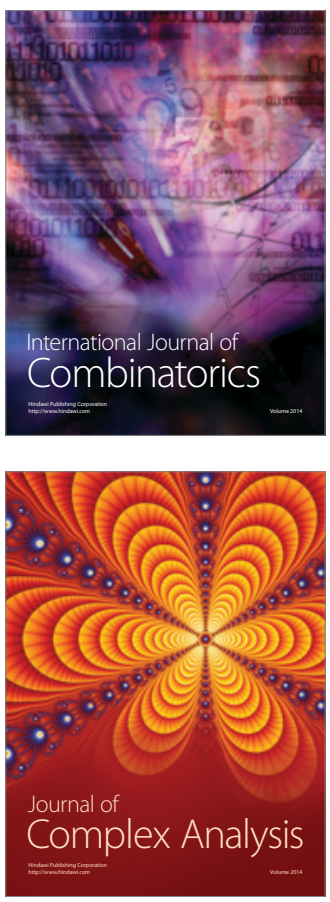

International Journal of

Mathematics and

Mathematical

Sciences
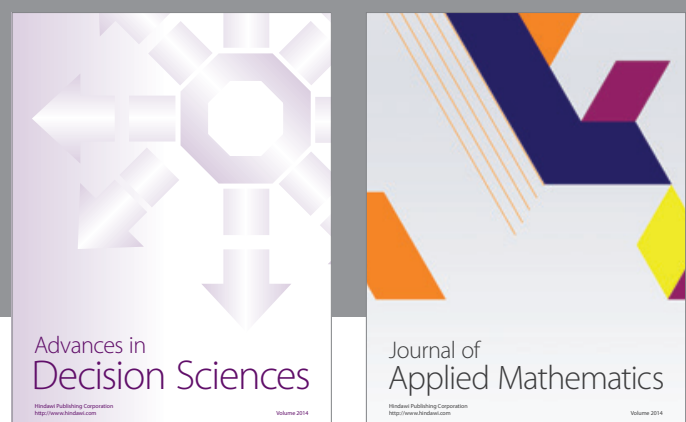

Journal of

Applied Mathematics
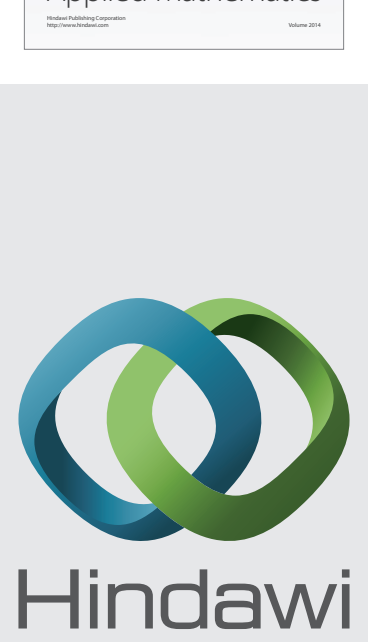

Submit your manuscripts at http://www.hindawi.com
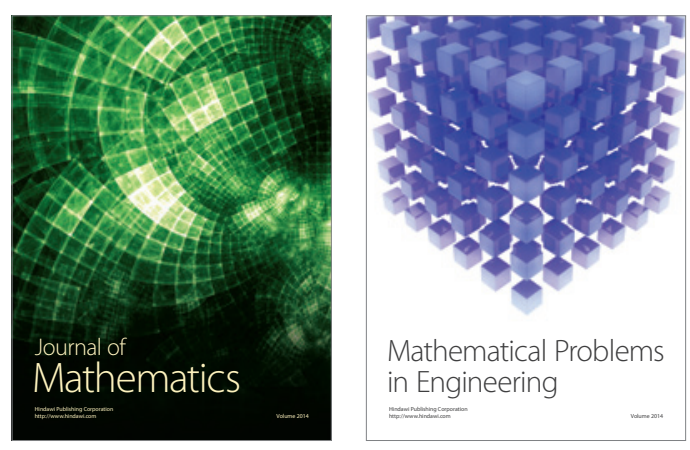

Mathematical Problems in Engineering
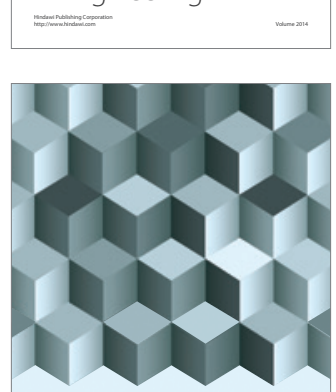

Journal of

Function Spaces
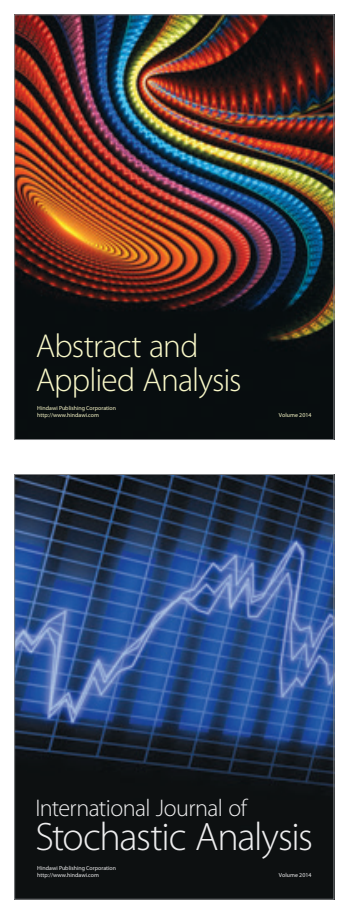

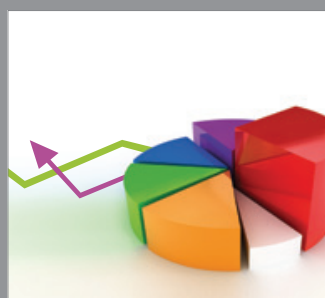

ournal of

Probability and Statistics

Promensencen
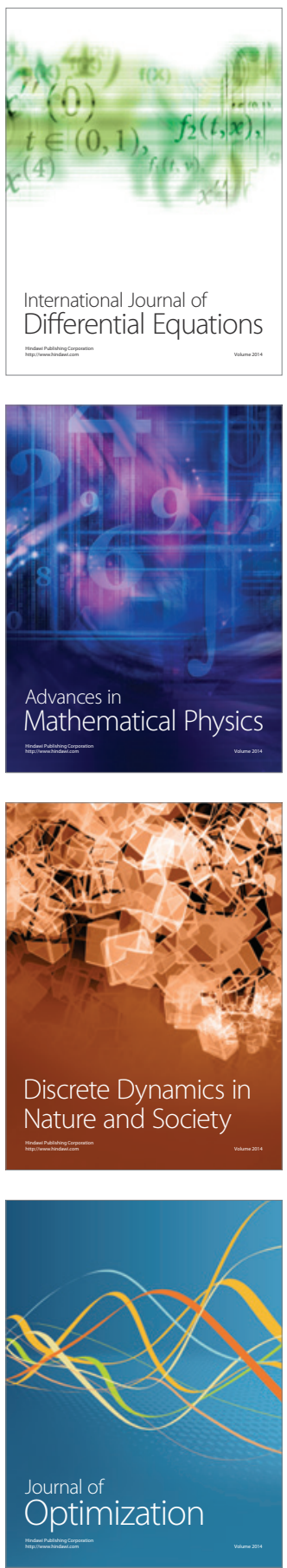